\title{
VR Binoculars: an immersive visualization framework for IoT data streams
}

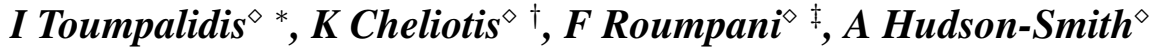 \\ $\diamond$ The Bartlett Centre for Advanced Spatial Analysis (CASA), University College London (UCL), UK \\ *ioannis.toumpalidis.16@ucl.ac.uk, ${ }^{\dagger}$ kostas.cheliotis.12@ucl.ac.uk, ${ }^{\ddagger}$.roumpani@ucl.ac.uk
}

Keywords: Virtual Reality, 3D, IoT, Urban Data, Visualisation

\begin{abstract}
Every day we find ourselves moving through a blend of material spaces and immaterial networks. This invisible layer created from the millions of the data streams and network connections that take place around us tends to get denser with the recent development and deployment of the IoT devices in the urban space. In our work we aim to explore how the available technology of Mixed Reality spectrum can be applied to provide us an immerse view of the information that exist within the invisible layer of the "cyberspace" [1]. To this extend, we propose "VR Binoculars", a digital visualization framework that operates in real time.The available Virtual (VR), Augmented (AR), and Mixed Reality (MR) technology is used as a medium to unveil the information that exist in our surrounding space. Specifically, we situate the user within an environment where the digital data visualizations and the physical space are matched together, providing to the user the ability to interact, orient themselves and navigate naturally.For us the proposed framework it is able to promote a better understanding of the IoT ecosystem, it can justify the use of sensors in the public space, and it can raise awareness about privacy and data sharing. Using different urban environments as test cases (indoor, outdoor) we present our methodology and our first results.
\end{abstract}

\section{IoT and data visualization}

The widespread use of IoT devices, with the constant capture, sharing, and retrieval of diverse data on multiple aspects of the urban environment around us taking place on user demand, or even automatically, has signalled a new era of the "Real-Time City" [2]. Billions of devices are estimated to be connected to the Internet within the next decade [3]. A new type of city infrastructures that include sensors, CCTVs, meters, transponders and several other systems are now being installed in large cities, providing an entirely new layer of information that needs to be integrated to a variety of monitoring, planning and decision making operations.As an example, the city of New York since 2015 has installed various of sensors in Times Square, such as Solar-powered wi-fi trashcans equipped with environmental sensors, CCTV's in combination with image recognition to monitor suspicious behavior and sensors in traffic lights to adjust circulation in real time [4]. In combination with mobile technology and social networks, these devices are constantly pushing information packages through established communication streams to larger ontologies for storage and analysis, giving form to a "wider embedded spatial intelligence" ecosystem [5].

The produced data ontologies offer an unrepresented spatial resolution data ontology [6] that can be reported back to the public domain through digital dashboards [7]. These systems, use sophisticated algorithms to mine, analyse and visualise a vast amount of live data, and condense them into a single two dimentional readable mash-up, usually displaying meters and graphs which update regularly [2]. However these visualizations don't always conform with the latest technologies in computer graphics and have limited capabilities to engage the end user. As a result more sophisticated solutions to enable the interaction between the citizens and the collected data streams are necessary [8]. Having as a main interest the urban domain, we focus to the spatial nature of these devices.

\section{The spatial nature of IoT}

IoT devices are strongly related to the physical space where they are located. Therefore drawing from the fundamentals of GIS, we can identify two primary characteristics for each IoT device, the data that each sensor is collecting,generating or transmitting and its physical location. It is noticeable that by their nature IoT present an interesting duality.A defined presence both in physical space (through the physical sensor's location), and in virtual space (or information space, as part of a bigger data ecosystem where it belongs). This point is further illustrated by Eno \& Thompson (2011) who highlight how the IoT has generated a more concrete link between information from the virtual world and objects in the physical world [9]. Ragget(2015) further suggests a framework for unifying the different IoT platforms, termed the "Web of Things", which would "enable worldwide discovery and interoperability" and further solidify the connections between real and virtual [10].

In outlining a vision for IoT, Gubbi et al. [11] highlight the need for data streams and services to be visualized in an attractive and easy to understand way, so as to maximize adoption. 
They go on to state that in addition to communicating content, an IoT visualization platform should also take advantage of meta-data which often include geo-location information. It is therefore of interest to not only maintain the physical-virtual aspect of IoT, but further highlight it, if the IoT platform is to be adopted and comprehended. Under this perspective, the IoT visualization problem might be examined as an instance of a spatial data visualization problem which in our work we try to tackle it using the emerging Mixed Reality technology.

\section{Research background}

\subsection{Fundamentals of Mixed Reality}

In 1999, one of the first definitions for Mixed Reality was given Milgram, Milgram, Colquhoun, et al. to supplement the existing broader views of Virtual Reality. The author introduces a graphical concept of two parallel continuums the Reality Virtuality (RV) Continuum and the Extent of World Knowledge Continuum (EWK) (Figure 1). Observing the parallel system, the Continuum goes from the Real Environment which is a world completely unmodeled (from the computer perspective) to the Virtual Environment were the world is completely modeled. Moving towards the center of the RV spectrum the uncertainty about the referencing point increases. Whenever we use the physical world to augment virtual objects on it,the author suggests the term of Augmented Reality (AR) whereas in the case were physical objects enhance a virtual environment, the author coins the term of Augmented Virtuality (AV) [12]. The entire continuum is also refereed as a Mixed Reality.

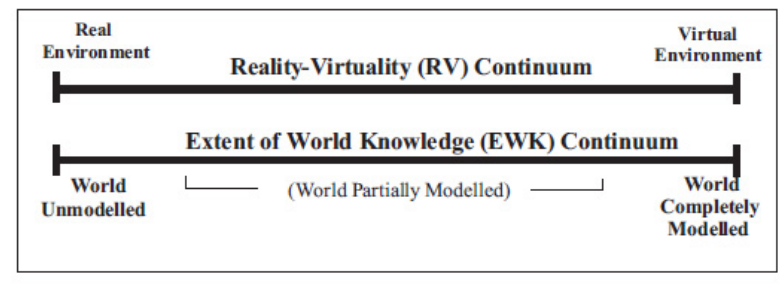

Fig. 1: RV Continuum, proposed by Milgram, Milgram, Colquhoun, et al. [12]

Based on this concept, we perceive mixed reality as not a discrete from AR and AV phase, but a state in between, that contains characteristics from both of them. Closer to the practical implementation of the MR application, Honig, Milanes, Scaria, et al. states that mixed reality system combine physical objects in at least one physical environment and virtual objects in at least on virtual environment. Furthermore the author highlights an application of this kind should run interactively in real time and be able to spatially map physical and virtual objects to each other [13]. Theoretically, the goal of a mixed reality application can be the creation of a "see-through" view of the real world. A process to record and display temporal information "indistinguishable from the the unmediated reality" [14].

\subsection{Virtuality for understanding physical reality}

The literature findings suggest that these "see through" windows towards the physical reality, tend to provide better understanding and awareness to the user for their surrounding space.

Perception via our body sensors is often the most concrete feature that enables us to interpret something as objectively real. This "factual knowledge" as Carbon is referring to it, is particularly strong when it comes to visual sense and even stronger with the implementation of haptical investigation [15]. Placing the user within a data rich environment, while the scale of the visualization remains unchanged compared to the physical world, users are able to discover new perspectives and connections in the data set [16] retaining the awareness and their "factual knowledge" about the physical space around them. To that extend, it is possible that the immerse mixed reality applications can provide effective alternatives to traditional visualization methods.

Research experiments measuring the effectiveness of VR applications in different scientific fields have started as early as 1995. In the field of tutoring and understanding Bliss and Tidwell researched the contribution of virtual reality training in the spatial navigation of a firefighters group [17]. Additionally, Johnston, Rae, Ariotti, et al. presented how VR applications help biology students to perceive and better understand microbes by bringing students and their observations in the same visual scale [16].

More related to our research field and the urban environment,Du, Zou, Shi, et al. investigated the real time visualization of BIM data in VR platforms enhance the communication and collaboration between partners aiming for a better decision making process [18]. To the same direction, Paes, Arantes, and Irizarry examined and measured the effectiveness of VR within the architecture, engineering and construction (AEC) industry highlighting the luck of similar quantitative researches in the years between 2005 and 2011 [19].

From a different point of view, Honig, Milanes, Scaria, et al. researched the use of VR spaces from a device perspective. In his work, Honig creates a hyperspace, "blurring the distinctive boundaries " between the virtual and the physical world. In this mixed reality space objects from both the physical and the virtual world are able to communicate in real time. The aim of this mixed reality application research is the creation of a co-operative virtual space where machines can work and be trained with safety [13].

It is noticeable that MR continuum can not only offer an environment dedicated for showcasing and visualizing generated data, but also it can be used as a test bench for the IoT prototypes. Towards this direction, the NVIDIA corporation presented the use of MR application as virtual labs where sensors for autonomous vehicles are brought to the test [20] pushing the boundaries of traditional simulation softwares.

In a broader conclusion Macias, Alvarez-Lozano, Estrada, et al. (2011) highlights that MR applications are by definition a 
fitting tool for visualizing IoT data as they combine elements from the physical and the virtual world. Therefore they are exceptionally good mediums for capturing the aforementioned duality of the IoT sensors that takes place in between the physical and informational space [21]

\subsection{Related Work}

Combinations of MR and IoT are seen in the theoretical work ofKodym (2012) who highlighted the potential to increase the efficiency of the information sharing process by linking information on physical objects (e.g. documentation of equipment), easily accessed through a VR interface [22]. More applied approaches proposed the development of learning environments [23] and workspaces that combine physical and virtual elements powered by IoT for industrial applications [24]. In the medical field, Fan et al.(2014) explored how an IoT-Based rehabilitation system for medical applications can be develop, and they suggest the use of VR and AR tools as a technique for facilitating human-machine interactions [25]. Incorporating geographic tools Lv et al.(2016) proposed a web-based, VR GIS platform for interacting with IoT in a Smart City context [26].

In the next section, we will present a proposal for visualizing and interacting with IoT in urban environments.

\section{Our proposed application}

\subsection{Aim of the project}

Taking into the account all of the aforementioned spatial characteristics of MR we propose a framework that we name "VR Binoculars". This mixed reality solution aims to visualize generated data that derive from various devices and sensors from the IoT ecosystem within the urban environment. Through this research project we aim to explore how mixed reality can be applied to achieve better understanding and additional insights for researchers, decision makers and operators.

We use the term of " binoculars" as we wanted to give emphasis on the fact that the entire digital visualization was build to match users' physical surroundings. To achieve this result we start by recreating a digital copy of the user's physical environment.This process combines 3D scan methodologies and manual 3D modeling techniques. Afterwards we apply the requested from the user data visualizations to this computer generated environment.Finally, during the run time of the application we continuously tracking the movement of user's head and hands, to give a kinesthetic sense to them.

Allowing the users to touch physical objects that co-exist in the virtual space, we provide a spatial anchor with the tangible reality helping them to orientate inside the virtual world and better perceive the information that is being displayed. We also have the ability to project a real time video feed that illustrates the space around them. The video can be generated from either an embedded or an external connected to the HMD camera.

\subsection{Proof-Of-Concept}

Towards the development of our proposed application we began by designing and implementing two Augmented Reality applications. These two applications were our first prototypes and were used as a supporting material to our proof of concept. The augmented reality technology was selected as an easier to access approach, compared to the fully immerse MR that we were aiming. In these experiments we used existing IoT sensors, actuators, and 3D models of our laboratory. The applications aimed to visualize data that were generated by the sensors in real time using commercial tracking technology (ARCore/ ARkit) or predefined traceable markers.

\subsubsection{Augmented Reality and weather data}

In this application we used the location-aware capabilities of Google Tango to place information in the form of 3D text at specific locations inside the office of our lab. The data were coinciding with a series of light-strip installations (Figure 2) that are linked with weather stations located to the building's roof.The development of the application was done in Unity, and the prototype demonstrated that the combination of physical and virtual spaces are indeed feasible with the commercially available tools.

\subsubsection{AR for low energy bluetooth sensors}

In the second test case we developed an Augmented Reality application for Android devices, which connects to a TI sensor tag (TexasIntrumets) via bluetooth and finds the location of the sensor via the 3D targets of the Vuforia Augmented Reality Software Development Kit (SDK) (Figure 3).

IoT has exploded with the use of Low Energy Bluetooth (BLE) devices as these sensors have enabled the connectivity between devices and the world around them using low energy consumption, which can last up to 4 years. BLE transceivers have reduced in size with chip scale packaging, making them small enough to easily fit alongside the various sensors in an IoT design [27].

\subsection{Developing the MR application}

The process for the development of the MR application can be divided in the following stages. Modeling, Matching and Operation.

For the modeling phase, we used Google Tango to 3D scan the interior space of the room where the VR Binoculars were planned to be installed. Afterwards the generated point cloud was manually edited and simplified to reduce its complexity and its file size. Complex meshes were substituted by primitive $3 \mathrm{D}$ objects as cubes, spheres, capsules etc. that can easier be transformed compared to their original meshes. To increase the feeling of realism in the final model, detailed 3D models of furnitures, lighting and textures were added. 

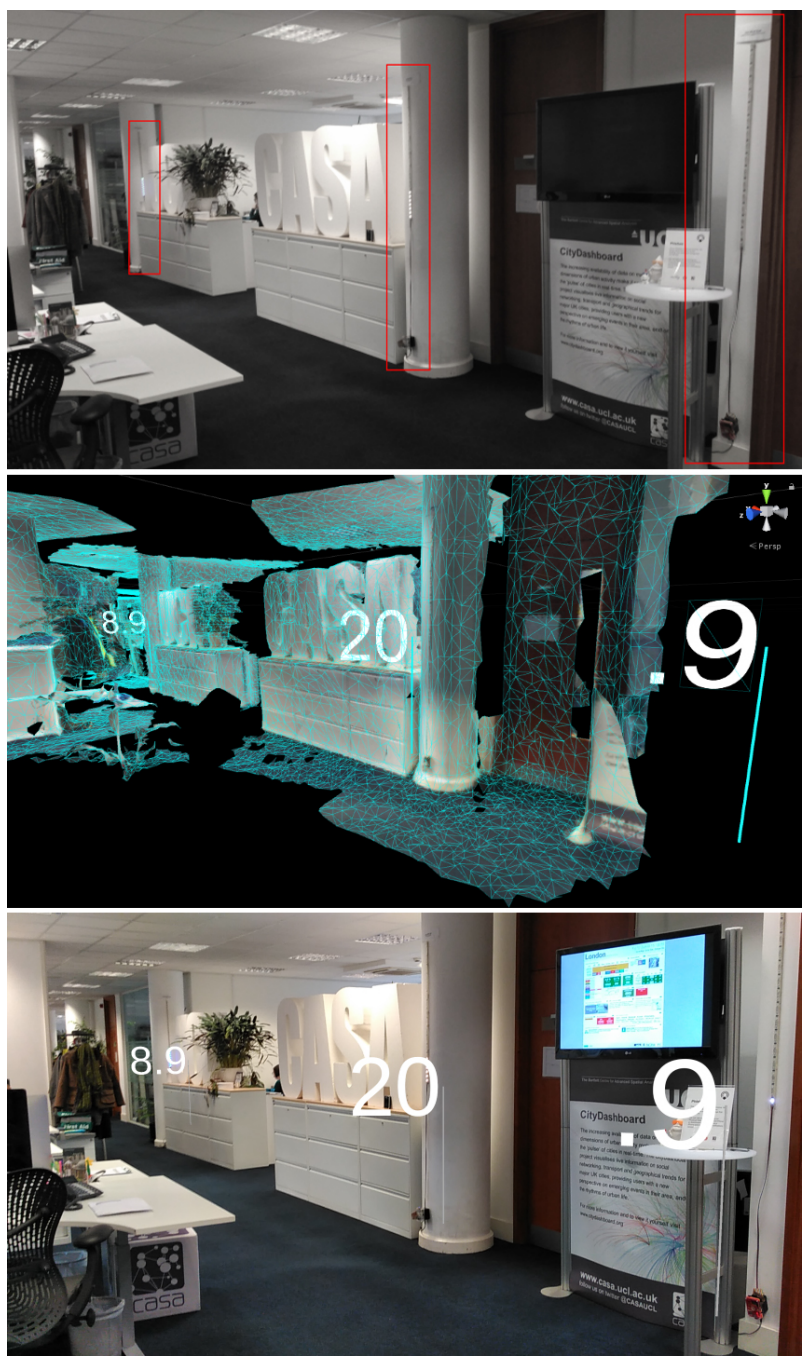

Fig. 2: Merging virtual and physical IoT. From top to bottom: actual environment, virtual reconstruction with sensor data readings incorporated, IoT data appended to physical objects. (Data overlaid to weather actuator lightstrips which were developed by Steven Gray)

After the modeling phase, the virtual environment needed to be calibrated and be matched with the viewer's physical location. This time consuming process was heavily based on the video stream that derives from the HTC VIVE's front camera and is visible within the virtual environment.

Finally during the last phase, a set of scripts were developed to enable interaction between user and the virtual elements. By tracking a physical controller we were able to design a virtual UI that gives a variety of different options to the user.

Some of the available options were the ability to activate different data layers, adjust the transparency of the surroundings, data input methods and selection capabilities 5 .
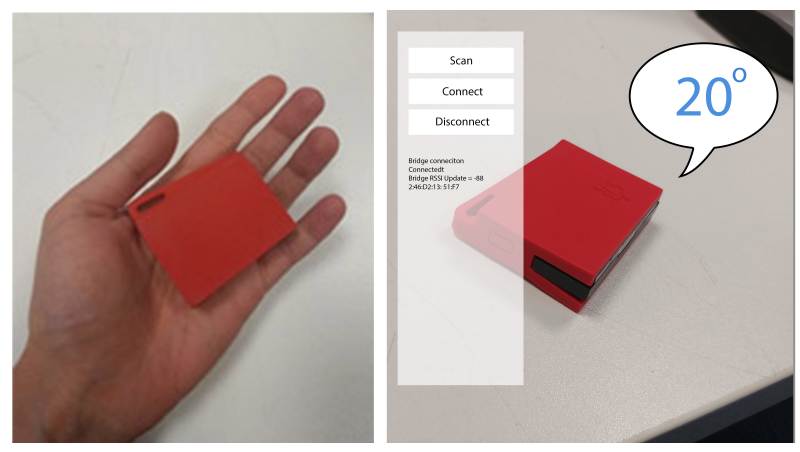

Fig. 3: TI sensor Tag reads in $A R$
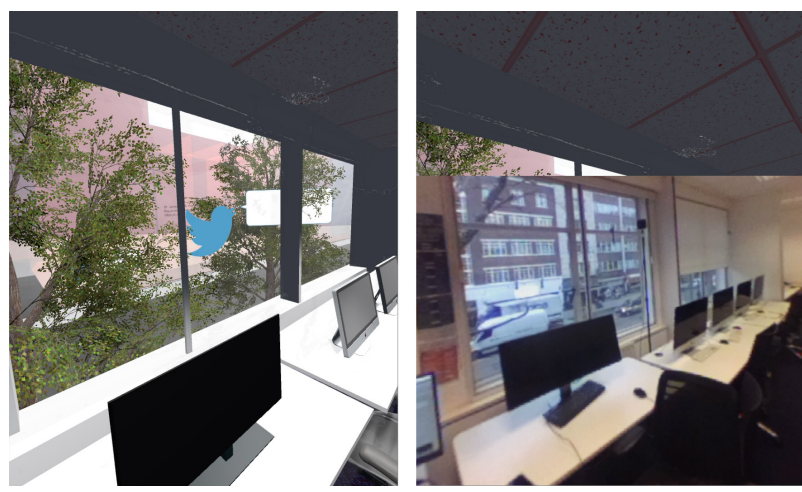

Fig. 4: Phases of the MR application development. Modeling (left) and Matching (right)

\subsection{Integrating social media data streams}

Experimenting with the integration of other supplementary data sets, we investigated the use of social media data. The invisible network of the IoT was supplemented by spatial related Twitter data. Specifically, real-time tweeter feeds were mined, analysed and visualised using the Twitter's "Stream API". Within the VR bionoculars solution we enabled the visualisation of nearby geo-located tweets, to create a virtual mash-up of the invisible networks that are generated around us. Tweets were tagged on the street network and were projected on the viewer's eye level.

\section{Results and discussion}

\subsection{Observations}

Although the current project is still on an early research stage, we were able to highlight some important to us characteristics for the use of MR applications in the domain of IoT data driven visualizations.

First of all, the available 3D Scanning solutions combined with the commercial VR head mounted displays (HMD), can deliver a very compelling and immersing experience to the end user. To our notice a solution that aims to visualize indoor sensors or devices that are located in a close proximity with the user can be benefited from the details of $3 \mathrm{~d}$ models and the kinesthetic 

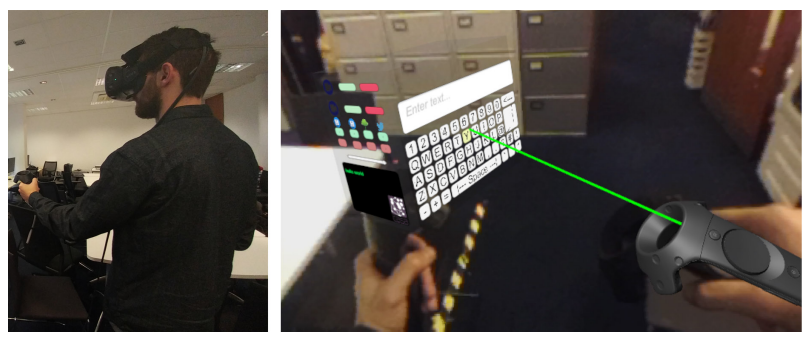

Fig. 5: Phases of the MR application development. Operation, Controls of a mixed reality

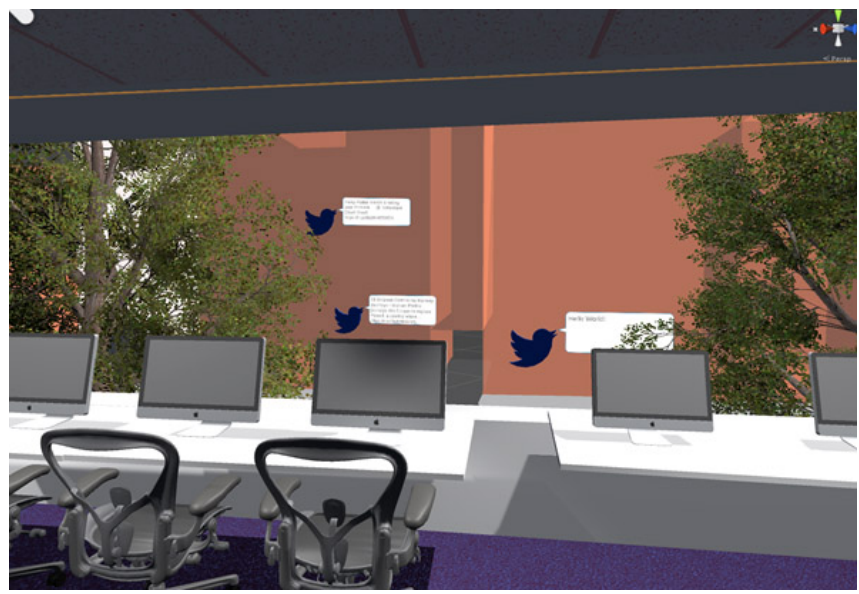

Fig. 6: Real-time tweet feeds in VR

experience. In contrast, open spaces or very complex environments need more thoughtful design considering the details that should be or not included.

Drawing from a test case of ours, visualizing a simulated dataset of connections between a number of stationary and movement devices in the Queen's Elisabeth Olympic Park, we came across a problem that relates with the visibility of the data. The clarity of the visualization changes dramatically according to the user's initial location. Even if we provide to the user a control of the transparency of various objects and buildings which sometimes might obstruct their view towards the data visualization, it is still difficult for them to have a clear view and an understanding of the data set. This problem, can possibly be overcome by giving an additional option to the user to move through the virtual space after the initialization.An option like this, can transform the point of view from an egocentric (first person point of view ) to an exocentric (third person or bird's view) within the Centricity continuum [12].

\subsection{Problems met}

During the development of the application we came across to some technical difficulties, that we wanted to present, by reflecting on the available literature findings.

Initial imposed by Milgram, Milgram, Colquhoun, et al. [12] there are two main problems during the development of a mixed reality application. First of all, is "the keyhole effect". This problem arises when the user is forced to see the physical space through a smaller compared to the normal field of view. Although the available technology surpasses by far Milgram's equipment in 1999, the problem still exists. In our research we used the HTC VIVE HMD which supports almost a 100 degrees of vertical and horizontal field of view, one of the largest FOV available in commercial products.

The second problem was the distortion of the video feed that was projected inside the virtual world during the calibration phase. As a result any view from a different angle than the one that the mounted camera had resulted to a relevant degree of distortion raising the awareness of a discontinuity phenomenon between the two environments [12]. In our case the front camera of the HMD generated a smaller in size, resolution and field of view video feed compared to the one that was available to be viewed through the HMD. Also,any attempt to project the camera feed in a smaller 2D plane destructed users' attention causing a parallax mismatch between the location of the physical objects and their virtual projections.

\section{Conclusions and future work}

Although our proposed application seems to mediate well enough the spatial attributes of the IoT devices, we still have to overcome many technical difficulties. Our first exploration, scratches only the surface of the capabilities that similar systems and applications might have in the future. Our next steps, include a quantitatively measurement of the efficiency of our application comparing our solution with other visualization methods.

Furthermore, we conceived our application as a part of a wider virtual framework that combines all different AR and VR applications in one integrated ecosystem. Our future goal for that system is to provide the ability for multiple users to engage with the urban IoT data streams, simultaneously, from different spatial locations and through a variety of devices. Towards this direction,our aim is to develop a MR application that will generate an active collaboration between citizens ,the research community and the stakeholders for the right and efficient use of IoT devices in the intra urban environment.

\section{Acknowledgments}

The research was supported by the ICRI Capstone project, a collaboration between Intel Labs Europe, University College London, Imperial College, and the Future Cities Catapult. The project demonstrated the ability of Internet of Things interventions to solve real urban challenges and was focused on Queen Elizabeth Olympic Park (QEOP) in East London. 


\section{References}

[1] M. Batty, "Virtual geography", Futures, vol. 29, no. 4-5, pp. 337-352, 1997, ISSN: 00163287. DOI: 10 . $1016 /$ S0016 - 3287(97) 00018 - 9. [Online]. Available: http : / / www . sciencedirect . com / science / article / pii / S0016328797000189 \% 5Cnhttps : / / www . sciencedirect . com / science / article/pii/s0016328797000189.

[2] R. Kitchin, "The real-time city? Big data and smart urbanism”, GeoJournal, vol. 79, no. 1, pp. 1-14, 2014, ISSN: 03432521. DOI: 10 . 1007 / s10708-0139516-8.

[3] J. Wakefield, "Why is that lamp-post watching me?", en-GB, BBC News, Sep. 2014. [Online]. Available: http : / / www . bbc . co . uk / news / technology-28159732 (visited on 01/15/2018).

[4] L. Poon, Next Time You Want Free Wi-Fi in NYC, Look For A Trash Can, en. [Online]. Available: http : / / www . citylab. com/ navigator/2015/07/ new - york - city - wi - fi - trash - cans / $398258 /$ (visited on 01/15/2018).

[5] N. Komninos, H. Schaffers, and M. Pallot, "Developing a Policy Roadmap for Smart Cities and the Future Internet", EChallenges e2011, pp. 1-8, 2011, ISSN: 0163-6804. DOI: 10.1109/MCOM.2013.6525605. [Online]. Available: http : / / www-sop . inria . fr/teams / axis / pages / bestpaper / 2011 eChallenges _ ref _ 196 - Roadmap - for Smart-Cities-Publised . pdf $\%$ Cnhttp : //www. urenio.org/wp-content/uploads/ 2008 / $11 / 2011$ - echallenges_ref_196Roadmap - for - Smart - Cities - Publised . pdf.

[6] R. Kitchin, "Big data and human geography: Opportunities, challenges and risks", Dialogues in human geography, vol. 3, no. 3, pp. 262-267, 2013.

[7] A. Hudson-Smith, "Tracking, tagging and scanning the city", Architectural Design, vol. 84, no. 1, pp. 40-47, 2014.

[8] B. Pokric, S. Krco, D. Drajic, M. Pokric, V. Rajs, Z. Mihajlovic, P. Knezevic, and D. Jovanovic, "Augmented reality enabled iot services for environmental monitoring utilising serious gaming concept", Journal of Wireless Mobile Networks, Ubiquitous Computing, and Dependable Applications, vol. 6, no. 1, pp. 37-55, 2015, ISSN: 20935382. [Online]. Available: http : / / www . scopus. com / inward / record. url ? eid $=2-$ s2.0-84926287740\&partnerID=tzotx3y1.

[9] J. D. Eno and C. Thompson, "Virtual and Real-World Ontology Services", IEEE Internet Computing, vol. 15, no. 5, pp. 46-52, Sep. 2011, ISSN: 1089-7801. DOI: 10 . $1109 /$ MIC.2011. 75.
[10] D. Raggett, "The Web of Things: Challenges and Opportunities", Computer, vol. 48, no. 5, pp. 26-32, May 2015, ISSN: 0018-9162. DOI: 10.1109 / MC . 2015 . 149.

[11] J. Gubbi, R. Buyya, S. Marusic, and M. Palaniswami, "Internet of Things (IoT): A vision, architectural elements, and future directions", Future Generation Computer Systems, 2013, ISSN: 0167-739X. DOI: 10 . $1016 /$ J.FUTURE.2013.01.010.

[12] P. Milgram, P. Milgram, H. Colquhoun, and H. Colquhoun, "A Taxonomy of Real and Virtual World Display Integration", Mixed Reality-Merging Real and Virtual Worlds, pp. 5-30, 1999. DOI: 10.1.1.32 . 6230.

[13] W. Honig, C. Milanes, L. Scaria, T. Phan, M. Bolas, and N. Ayanian, "Mixed reality for robotics", IEEE International Conference on Intelligent Robots and Systems, vol. 2015-Decem, pp. 5382-5387, 2015, ISSN: 21530866. DOI: 10.1109 /IROS . 2015.7354138.

[14] C.-C. Carbon, "The first 100 milliseconds of a face: on the microgenesis of early face processing.", Perceptual and Motor Skills, vol. 113, no. 3, pp. 859-874, 2011, ISSN: 0031-5125. DOI: $10.2466 / 07.17 .22$. PMS . $113.6 .859-874$. [Online]. Available: http : / / www . amsciepub . com/doi / abs / 10 . 2466 / $07.17 .22 . \mathrm{PMS} .113 .6 .859-874 \div 5$ Cnhttp: //dx.doi.org/10.2466/07.17.22. PMS . $113.6 .859-874$.

[15] _ - "Understanding human perception by humanmade illusions", Frontiers in Human Neuroscience, vol. 8, no. July, pp. 1-6, 2014, ISSN: 1662-5161. DOI: 10.3389 / fnhum. 2014.00566. [Online]. Available: http : / / journal . frontiersin . org / article/10.3389/fnhum.2014.00566/ abstract.

[16] A. P. Johnston, J. Rae, N. Ariotti, B. Bailey, A. Lija, R. Webb, C. Ferguson, S. Maher, T. P. Davis, R. I. Webb, J. Mcghee, and R. G. Parton, "Journey to the centre of the cell: Virtual reality immersion into scientific data", Traffic, no. September, pp. 1-6, 2017, ISSN: 16000854. DOI: $10.1111 /$ tra.12538.

[17] J. P. Bliss and P. D. Tidwell, "The Effectiveness of Virtual Reality for Administering Spatial Navigation Training to Police Officers", Proceedings of the Human Factors and Ergonomics Society Annual Meeting, vol. 39, no. 14, pp. 936-936, 1995, ISSN: 1541-9312. DOI: 10 . $1177 / 154193129503901412$. [Online]. Available: http: / / journals. sagepub. com/doi / 10 . $1177 / 154193129503901412$.

[18] J. Du, Z. Zou, Y. Shi, and D. Zhao, "Zero latency: Real-time synchronization of BIM data in virtual reality for collaborative decision-making", Automation in Construction, vol. 85, no. August 2016, pp. 51-64, 2018, ISSN: 09265805. DOI: $10.1016 / j$. autcon. 2017 . 
10.009. [Online]. Available: https: / / doi .org/ $10.1016 / j$.autcon.2017.10.009.

[19] D. Paes, E. Arantes, and J. Irizarry, "Immersive environment for improving the understanding of architectural 3D models: Comparing user spatial perception between immersive and traditional virtual reality systems", $A u$ tomation in Construction, vol. 84, no. August 2016, pp. 292-303, 2017, ISSN: 09265805. DOI: 10 . 1016 / j. autcon . 2017.09.016. [Online]. Available: http://dx.doi.org/10.1016/j.autcon . 2017.09 .016 .

[20] Welcome to the Holodeck, NVIDIA's Design Lab of the Future - NVIDIA Newsroom, 2017. [Online]. Available: https : / / nvidianews . nvidia . com / news / welcome-to-the-holodeck-nvidias-design-lab-of-the-future.

[21] J. A. G. Macias, J. Alvarez-Lozano, P. Estrada, and E. A. Lopez, "Browsing the Internet of Things with Sentient Visors", Computer, vol. 44, no. 5, pp. 46-52, May 2011, ISSN: 0018-9162. DOI: 10.1109/MC.2011.128.

[22] O. Kodym, "Creation of Virtual Reality space based on Internet of Things", in Proceedings of the 13th International Carpathian Control Conference (ICCC), May 2012, pp. 296-299. DOI: 10 . 1109 / CarpathianCC.2012.6228657.

[23] A. Peña-Ríos, V. Callaghan, M. Gardner, and M. J. Alhaddad, "Remote mixed reality collaborative laboratory activities: Learning activities within the InterReality portal", in Proceedings of the 2012 IEEE/WIC/ACM International Conference on Web Intelligence and Intelligent Agent Technology Workshops, WI-IAT 2012, 2012, ISBN: 9780769548807. DOI: 10 . 1109 / WI IAT. 2012.43.

[24] P. Galambos, P. Baranyi, and I. J. Rudas, "Merged Physical and Virtual Reality in Collaborative Virtual Workspaces: the VirCA Approach",

[25] Y. J. Fan, Y. H. Yin, L. D. Xu, Y. Zeng, and F. Wu, "IoTBased Smart Rehabilitation System", IEEE Transactions on Industrial Informatics, vol. 10, no. 2, pp. 15681577, May 2014, ISSN: 1551-3203. DOI: $10.1109 /$ TII.2014.2302583.

[26] Z. Lv, T. Yin, X. Zhang, H. Song, and G. Chen, "Virtual Reality Smart City Based on WebVRGIS", IEEE Internet of Things Journal, vol. 3, no. 6, pp. 1015-1024, Dec. 2016, ISSN: 2327-4662. DOI: $10.1109 /$ JIOT . 2016.2546307.

[27] European Editors, Bluetooth Low Energy Wearable Sensors IoT - DigiKey, 2017. [Online]. Available: https : / / www . digikey - co . uk / en / articles / techzone / 2017 / may / using-bluetooth-low-energy-connectwearable-wireless-sensors-iot. 